\title{
Significant expression of CHK1 and p53 in bladder urothelial carcinoma as potential therapeutic targets and prognosis
}

\author{
LINFENG ZHENG $^{1 *}$, YUPING ZHU $^{2 *}$, LEI LEI $^{3}$, WENYONG SUN $^{1}$, GUOPING CHENG $^{1}$ and SHIFENG YANG ${ }^{1}$ \\ Departments of ${ }^{1}$ Pathology, ${ }^{2}$ Colorectal Surgery and ${ }^{3}$ Medical Oncology, Zhejiang Cancer Hospital, \\ Hangzhou, Zhejiang 310022, P.R. China
}

Received April 19, 2017; Accepted October 25, 2017

DOI: $10.3892 / \mathrm{ol} .2017 .7344$

\begin{abstract}
Checkpoint kinase 1 (CHK1) and p53 are involved in cell-cycle checkpoint, and cellular response to DNA damage. CHK1 and p53 are overexpressed in bladder urothelial carcinoma (BUC); however, a clear elucidation on their interaction and influence in the progress of BUC is absent. The aim of the present study was to examine the correlation between CHK1 and p53 in BUC, and analyze their value as therapeutic targets and prognostic indicators in BUC. A clinically annotated cohort of 110 patients with BUC was identified retrospectively. EnVision-based immunohistochemistry and western blot analysis of the aforementioned DNA repair proteins were conducted on formalin-fixed-paraffin-emb edded or frozen tissues from the primary tumor. A total of 45 peritumoral tissue cases were assessed similarly as the control group. In the cohort of 110 patients with BUC, a significant overexpression of CHK1 and p53 was observed in primary compared with the peritumoral tissues $(\mathrm{P}<0.05)$. CHK1 and p53 demonstrated a positive correlation in BUC, and both were positively associated with the histological grade, clinical pathological staging, lymphatic metastasis and the 5 -year survival rate $(\mathrm{P}<0.05)$. However, $\mathrm{CHK} 1$ and $\mathrm{p} 53$ were not associated with sex, age, tumor diameter, single/multiple sites or incipient/recurrence. The overexpression of CHK1 and p53, and their synergistic interaction were putatively correlated with the physiology of BUC that may be deemed as potential therapeutic targets and prognostic indicators.
\end{abstract}

Correspondence to: Dr Lei Lei, Department of Medical Oncology, Zhejiang Cancer Hospital, 1 East Road of Banshan, Hangzhou, Zhejiang 310022, P.R. China

E-mail: leilei@zjcc.org.cn

Dr Wenyong Sun, Department of Pathology, Zhejiang Cancer Hospital, 1 East Road of Banshan, Hangzhou, Zhejiang 310022, P.R. China

E-mail: sunwy@zjcc.org.cn

${ }^{*}$ Contributed equally

Key words: bladder urothelial carcinoma, CHK1, p53, therapy targets, prognosis

\section{Introduction}

Bladder urothelial carcinoma (BUC) is a common malignant bladder cancer, the fourth leading cause of cancer in males, accounting for $\sim 4 \%$ of the cancer-related deaths (1). Despite the surgical treatment of transurethral resection (TUR) and postoperative recovery pathways, the incidence of bladder recurrence within 5 years could be up to $20-75 \%$ worldwide (2). BUC is staged via the tumor-node-metastasis (TNM) system, which describes the degree of invasion (Tis-T4) (3). Approximately, 75\% of the BUC patients present non-muscle-invasive bladder cancer (NMIBC; stage Ta-1). Surgical removal by TUR of bladder tumor is still the major treatment of NMIBC; yet, half of NMIBC patients treated with TUR have a recurrence of the disease and 5-25\% progressed to muscle-invasive bladder cancer (MIBC, stage T2 and above) after repeated recurrences (4). Patients with MIBC are at high risk of local invasion and distant metastasis, despite radical cystectomy and pelvic lymph-node dissection, up to $50 \%$ of patients still develop tumours at distant sites and exhibit a less favorable prognosis with a 5-year survival of $<50 \%(5,6)$. Hitherto, a number of factors have been reported as potential targets and/or prognostic markers, such as MAP4K1, Karyopherin $\alpha 2$ (KPNA2), thymosin $\beta 4$ (T $\beta 4)$, Her2/neu, and microRNAs (7-12). However, substantiated pathological or clinical tests to predict the response are yet lacking. Herein, we sought to identify the responsive factors, including cell cycle checkpoint kinase 1 (CHK1) and p53, which are reported less in BUC with respect to their advantages.

CHK1 is an enzyme, essential for preventing mitosis in response to DNA damage, primarily responding to replication fork interference in the S-phase and DNA damage in the G2 phase $(13,14)$. CHK1 kinase regulates the cell cycle progression from $\mathrm{S}$ to $\mathrm{M}$ phase following disruption of DNA replication or some types of DNA damage and has also been reported to play a role in the $S$ phase in undisturbed cells $(15,16)$. Some studies indicated that CHK1-deficient tumor cells exhibit multiple defects, such as the loss of response to cell cycle checkpoint arrest, retarded cell proliferation, and increased sensitivity to DNA-damaging agents $(17,18)$. Thus, understanding the status of the pathways is crucial for effective targeted therapies against the progression of BUC.

$\mathrm{p} 53$, one of the major tumor suppressors, similar to CHK1, exerts several mechanisms underlying the anticancer function 
and also plays a role in apoptosis, genomic stability, and inhibition of angiogenesis (19). p53 occurs as wild-type and mutant isoforms. The antitumor activity of wild-type p53 primarily enabling the DNA-damaged cells into G1/G0 arrest and DNA repair process before entering the $\mathrm{S}$ phase in anti-cancer mechanisms is carried out by i) activating the DNA repair proteins when DNA has sustained damage; ii) arresting the cell growth by stalling the cell cycle at the G1/S checkpoint upon recognition of DNA damage; iii) initiating apoptosis if DNA damage proves to be irreparable $(20,21)$. On the other hand, the mutant p53 loses its antitumor function and induces abnormal gene expression, thereby leading to tumor progression $(21,22)$. Although many reports described an abnormal p53 in BUC, whether it could serve as a prognostic factor in BUC is still unclear.

In the study, we examined CHK1 and p53 in BUC specimens and peritumoral tissues, investigated their expression and interaction in different histological grades, clinical pathological staging, and 5-year survival rate. In addition, we also assessed their value as potential therapeutic targets and for prognosis in BUC.

\section{Materials and methods}

Specimens and clinical data. A total of 110 specimens of bladder cancer and 45 peritumoral bladder tissues (the adjacent normal tissues $>2 \mathrm{~cm}$ from the cancer tissue) were collected between 2009 and 2014 at the Zhejiang Cancer Hospital (Zhejiang, China). The detailed clinicopathological data including age, sex, TNM stage, histological subtype and grade, tumor diameter, single/multiple sites, and patients with incipience/recurrence were assimilated (Table I). Tumor grades were determined based on the 2016 World Health Organization/International Society of Urologic Pathology classification (23); the pathologic stage was assigned according to the 2010 American Joint Committee on Cancer 7 th TNM staging system (24). The specimens were frozen at $-80^{\circ} \mathrm{C}$ or fixed in $10 \%$ formalin, embedded in paraffin, sliced continually, and subjected to hematoxylin and eosin (H\&E) and immunohistochemistry (IHC) staining. This reterospective study has been approved by the Ethics Committee of the Zhejiang Cancer Hospital.

IHC. Mouse anti-human monoclonal antibodies against CHK1 and p53 were purchased from Santa Cruz Biotechnology (Santa Cruz, CA, USA). IHC EnVision method was performed as follows: $4 \mu \mathrm{m}$ paraffin-embedded tissue sections were mounted on glass slides. After deparaffinization in xylene, the slides were immersed in a target retrieval buffer solution; subsequently, the slides were treated with EDTA buffer ( $\mathrm{pH} 9.0)$ bath. The endogenous peroxidase was blocked by incubation in methanol containing $0.3 \% \mathrm{H}_{2} \mathrm{O}_{2}$ for $30 \mathrm{~min}$. IHC staining was performed using the EnVision System (EnVision'; Dako, Carpentaria, CA, USA). The slides were incubated overnight at $4^{\circ} \mathrm{C}$ with primary antibody against $\mathrm{CHK} 1$ and p53. followed by immersion in diaminobenzidine (DAB) for signal visualization. The negative control slides were probed with phosphate buffered saline (PBS) instead of the primary antibody.

Western blot analysis. Total protein from BUC or normal tissues was extracted using RIPA buffer containing the protease inhibitor PMSF (Bocai Bio Company, Shanghai, China) and quantified by bicinchoninic acid (BCA) method (Pierce, Rockford, IL, USA). The protein samples were resolved on $8 \%$ SDS-polyacrylamide gel and transferred to PVDF membrane (Amersham, Buckinghamshire, UK). The membranes were blocked with $5 \%$ dry milk for $1 \mathrm{~h}$ in Tris-buffered saline and 0.1\% Tween-20 (TBS/Tween-20) (Dako). After washing in TBS/Tween-20, the membranes were probed with rabbit polyclonal CHK1 $(1: 2,000)$, or mouse monoclonal p53 (1:2,000), or mouse monoclonal GAPDH (1:5,000; all Abcam, Cambridge, UK) antibodies overnight at $4^{\circ} \mathrm{C}$. After washing with TBS/Tween-20, the membranes were probed with HRP-conjugated rabbit anti-mouse or goat anti-rabbit $\operatorname{IgG}$ (1:5,000; Abcam) for $1 \mathrm{~h}$ at room temperature and washed with TBS/Tween-20. The immunoreactive bands on the membrane were detected using ECL Plus ${ }^{\mathrm{TM}}$ Western Blotting Detection Reagents (Amersham). The signal of western blotting band was quantified using gray-scale analysis software and data was normalized to GAPDH.

Determination of results. CHK1 is expressed mainly in the cytoplasm, also slightly in the nucleus, whereas p53 is primarily expressed in the nucleus. Semi-quantitative results were determined according to the percentage and intensity of the staining pattern of positive cells: i) According to the percentage of positive cells in the total counted cells, the scores were divided into 4 levels: 0 , positive cells $<10 \%$; 1 , positive cells moderate $10-24 \%$; 2 , positive cells moderate $25-49 \%$; 3 , positive cells moderate $50-74 \%$; 4 , positive cells $>75 \%$. ii) According to the intensity of the staining pattern, the scores were ascribed as follows: 0, colorless; 1, yellow; 2, brown; 3 , tan. The cell staining was comprehensively judged based on the multiplication of scores of the positive cells and the intensity of staining: $0-1$, negative; $\geq 2$, positive.

Statistical methods. SPSS 21.0 (IBM SPSS, Armonk, NY, USA) was used to analyze the data and determine the correlations among various parameters. The chi-square and Fisher's exact tests were applied to determine the statistical significance, and Spearman's rank correlation test was employed for determining the relationship among CHK1 and p53 in BUC. Kaplan-Meier survival analysis and log-rank test were used for determining the survival curves. $\mathrm{P}<0.05$ was considered to indicate a statistically significant difference.

\section{Results}

CHK1 and p53 are expressed significantly in BUC than in peritumoral tissue, showing a large difference between Ta-T1 and T2-T4 groups. The positive rates of CHK1 in BUC and peritumoral tissues were $73.6 \%(81 / 110)$ and $6.7 \%(3 / 45)$, respectively, and that of p53 were $52.7 \%$ (58/110) and $2.2 \%$ (1/45), respectively. The differences were statistically significant $(\mathrm{P}<0.05)$ (Table II). IHC demonstrated that CHK1 and p53 were expressed significantly in both Ta-T1 and T2-T4 groups than the normal group. In addition, CHK1 and p53 were stained deeply in T2-T4 than in Ta-T1 group, indicating significant differences. A total of 110 cases were checked and the representative pictures were shown in (Fig. 1). For further 
Table I. Clinicopathological characteristics of BUC patients.

\begin{tabular}{|c|c|c|c|c|c|c|c|}
\hline Characteristic & $\mathrm{N}$ & $\begin{array}{l}\text { CHK1-positive } \\
\text { cases }(\%)\end{array}$ & $\chi^{2}$-value & P-value & $\begin{array}{l}\text { p53-positive } \\
\text { cases }(\%)\end{array}$ & $\chi^{2}$-value & P-value \\
\hline \multicolumn{8}{|l|}{ Sex } \\
\hline Male & 94 & $70(74.5)$ & \multirow[t]{2}{*}{0.41} & \multirow[t]{2}{*}{0.63} & $49(43.6)$ & \multirow[t]{2}{*}{0.002} & \multirow[t]{2}{*}{0.96} \\
\hline Female & 16 & $11(68.5)$ & & & $9(50.0)$ & & \\
\hline \multicolumn{8}{|l|}{ Age, years } \\
\hline$<63$ & 48 & $35(72.9)$ & \multirow[t]{2}{*}{1.17} & \multirow[t]{2}{*}{0.27} & $20(41.7)$ & \multirow[t]{2}{*}{2.40} & \multirow[t]{2}{*}{0.12} \\
\hline$\geq 63$ & 62 & $46(74.2)$ & & & $38(61.3)$ & & \\
\hline \multicolumn{8}{|l|}{ Histologic grade } \\
\hline High grade & 47 & $31(66.0)$ & \multirow[t]{2}{*}{7.54} & \multirow[t]{2}{*}{0.006} & $23(48.9)$ & \multirow[t]{2}{*}{4.45} & \multirow[t]{2}{*}{0.016} \\
\hline Low grade & 63 & $50(79.3)$ & & & $35(55.6)$ & & \\
\hline \multicolumn{8}{|l|}{ Pathologic T } \\
\hline Ta-T1 & 50 & $32(64.0)$ & \multirow[t]{2}{*}{4.54} & \multirow[t]{2}{*}{0.032} & $17(34.0)$ & \multirow[t]{2}{*}{5.46} & \multirow[t]{2}{*}{0.019} \\
\hline $\mathrm{T} 2-\mathrm{T} 4$ & 60 & $45(72.3)$ & & & $36(60.0)$ & & \\
\hline \multicolumn{8}{|c|}{ Lymphatic metastasis } \\
\hline Positive & 51 & $42(82.4)$ & \multirow[t]{2}{*}{5.07} & \multirow[t]{2}{*}{0.024} & $36(70.1)$ & \multirow[t]{2}{*}{4.89} & \multirow[t]{2}{*}{0.027} \\
\hline Negative & 59 & $39(66.1)$ & & & $22(37.3)$ & & \\
\hline \multicolumn{8}{|c|}{ Tumor diameter, $\mathrm{cm}$} \\
\hline$<2$ & 18 & $11(61.1)$ & \multirow[t]{2}{*}{0.002} & \multirow[t]{2}{*}{0.96} & 7 (38.9) & \multirow[t]{2}{*}{0.12} & \multirow[t]{2}{*}{0.65} \\
\hline$\geq 2$ & 92 & $70(76.1)$ & & & $51(55.4)$ & & \\
\hline \multicolumn{8}{|c|}{ Numbers of tumor } \\
\hline Single & 47 & $34(72.3)$ & \multirow[t]{2}{*}{0.003} & \multirow[t]{2}{*}{0.82} & $21(44.9)$ & 1.28 & 0.27 \\
\hline Multiple & 64 & $47(73.4)$ & & & $37(57.9)$ & & \\
\hline Tumor formatio & & & & & & & \\
\hline No recurrence & 78 & $63(80.8)$ & 0.44 & 0.51 & $45(57.8)$ & 0.27 & 0.61 \\
\hline Recurrence & 32 & $18(56.3)$ & & & $13(40.6)$ & & \\
\hline
\end{tabular}

BUC, bladder urothelial carcinoma; CHK1, checkpoint kinase 1.

Table II. Expression of CHK1 and p53 proteins in the normal or BUC tissues.

\begin{tabular}{|c|c|c|c|c|c|c|c|}
\hline Group & $\begin{array}{c}\text { Total } \\
\text { number }\end{array}$ & $\begin{array}{l}\text { CHK1-positive } \\
\text { cases }(\%)\end{array}$ & $\chi^{2}$-value & P-value & $\begin{array}{l}\text { p53-positive } \\
\text { cases }(\%)\end{array}$ & $\chi^{2}$-value & $\mathrm{P}$-value \\
\hline Normal & 45 & $3(6.7)$ & 6.53 & 0.011 & $1(2.2)$ & 7.03 & 0.008 \\
\hline BUC & 110 & $81(73.6)$ & & & $58(52.7)$ & & \\
\hline Ta-T1 & 50 & $32(64.0)$ & 4.54 & 0.032 & $17(34.0)$ & 5.46 & 0.019 \\
\hline $\mathrm{T} 2-\mathrm{T} 4$ & 60 & $49(81.7)$ & & & $41(68.3)$ & & \\
\hline
\end{tabular}

BUC, bladder urothelial carcinoma; CHK1, Checkpoint kinase 1.

Table III. Correlation between CHK1 and p53 in BUC.

\begin{tabular}{ccccc}
\hline & \multicolumn{2}{c}{ CHK1 } & & \\
\cline { 2 - 3 } $\mathrm{p} 53$ & + & - & $\chi^{2}$-value & P-value \\
\hline+ & 48 & 10 & 5.259 & 0.022 \\
- & 33 & 19 & & \\
\hline
\end{tabular}

$\mathrm{r}=0.480$. BUC, bladder urothelial carcinoma; CHK1, checkpoint kinase 1. assessment, we examined protein expression of four cases in each group by western blot analysis, the representative bands were selected to be demonstrated; and the intensity of the immunoreactive bands markedly supported the previous results (Fig. 2).

Expressions of CHK1 and p53 proteins are related with clinical pathological stage, histological grade, and lymphatic metastasis in BUC. We demonstrated that the positive rate of CHIK1 in T2-T4 (81.7\%) was higher than in Ta-T1 period 

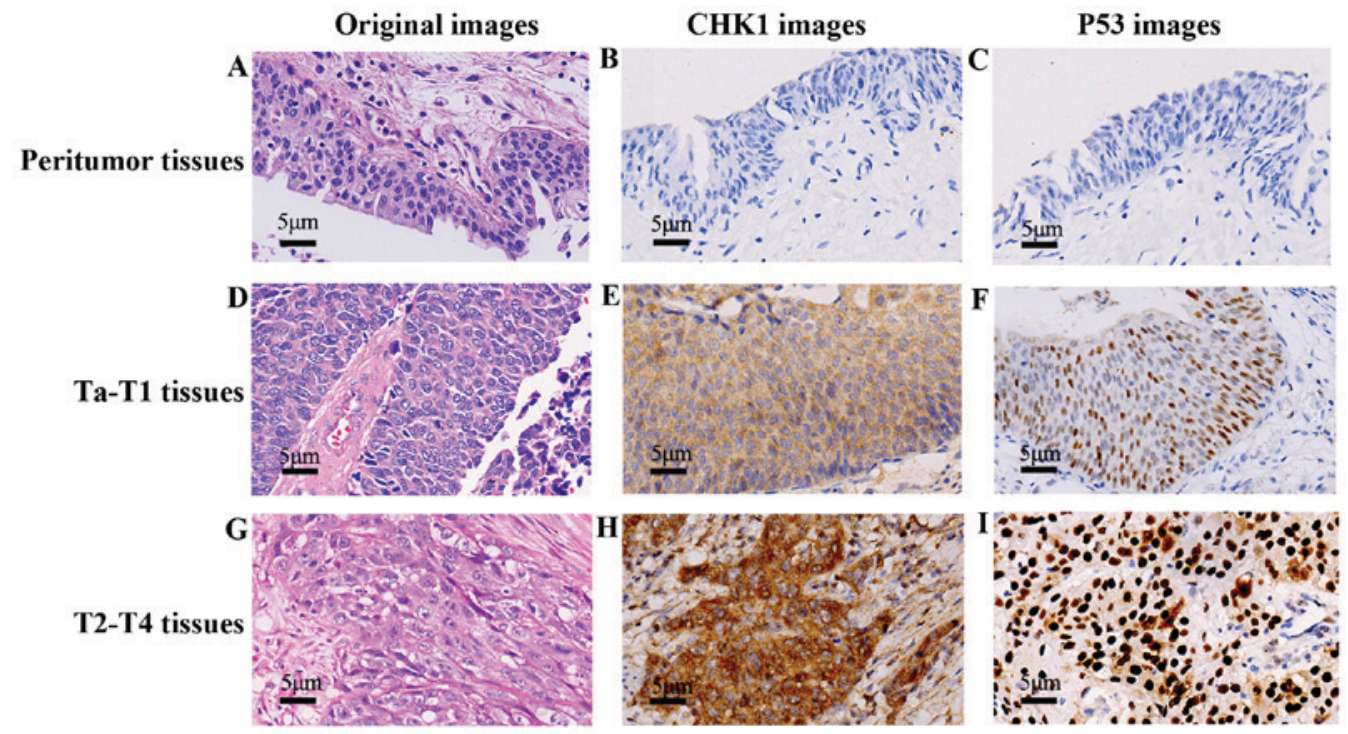

Figure 1. CHK1 and p53 expression profiles in peritumoral, Ta-T1, and T2-T4 BUC tissues, respectively. A total of 110 cases were checked in immunohistochemistry EnVision method (x400), and the representative pictures were shown. In the peritumoral group, we checked the H\&E staining (A) IHC staining with CHK1 (B) and p53 (C) respectively, both the CHK1 and p53 displayed a negative expression. In Ta-T1 group, H\&E staining showed atypical tumor cells (e.g., large, irregular and partial nucleus) (D) IHC staining with CHK1 (E) and p53 (F) were positive, the location and depth of color were preponderant than the peritumoral group (B and C). In T2-T4 group, H\&E staining displayed cancer nests (G) CHK1 (H) and p53 (I) in IHC were stained intensely than the Ta-T1 group (E and $\mathrm{F})$.

$(64.0 \%)$ and that of $\mathrm{p} 53$ was $68.3 \%$ in $\mathrm{T} 2-\mathrm{T} 4$ and $34.0 \%$ in Ta-T1 BUC. In the histological grade, CHK1 occupied 79.3 and $66 \%$ in the low and high grade, respectively, whereas p53 was $55.6 \%$ in low grade and $48.9 \%$ in high. Furthermore, in 51 cases with lymphatic metastasis, $\mathrm{CHK} 1$ showed a positive rate of $82.4 \%$, whereas p53 was $70.3 \%$. All the data were statistically significant $(\mathrm{P}<0.05)$. However, the protein expression did not correlate with sex, age, tumor diameter, single/multiple sites, and patients with incipience/recurrence (Tables I, II).

Positive correlation of $\mathrm{CHK} 1$ and 553 expressions in $B U C$. The positive correlation between CHK1 and p53 ( $\mathrm{r}=0.480$, $\mathrm{P}<0.05$ ) demonstrated a synergistic influence in BUC development (Table III).

CHK1 and p53 as potential prognostic factors for BUC. In the cohort of 110 BUC cases, the shortest survival time was 7 months and the longest was 82 months, with an average of 55.16 months and median 53 months. Kaplan-Meier and the log-rank test analyses showed that the overall 5-year survival rate was $62.7 \%(67 / 110)$. The 5 -year survival rate of CHK1-positive and negative cases was 56.8 and $75.0 \%$, respectively, with a significant difference $\left(\chi^{2}=6.98, \mathrm{P}=0.008\right)$ (Fig. 3). With respect to p53, the 5-year survival rate of positive and negative cases was 47.7 and $72.7 \%$, respectively $\left(\chi^{2}=7.63, \mathrm{P}=0.006\right)$ (Fig. $\left.3 \mathrm{~B}\right)$. In further, we divided the cohort into NMIBC (Ta-1 in pathologic, 50 cases) and MIBC (T2-4 in pathologic, 60 cases) groups, and further in sub-groups according the markers: CHK1 positive and p53 positive (NMIBC, 8 cases; MIBC, 27 cases); CHK1 positive and p53 negative (NMIBC, 24 cases; MIBC, 18 cases); CHK1 negative and p53 positive (NMIBC, 9 cases; MIBC,9 cases); CHK1 negative and p53 negative (NMIBC, 9 cases; MIBC, 6 cases). The data in NMIBC group indicated that CHK1 negative and p53 negative sub-group had obvious advantage
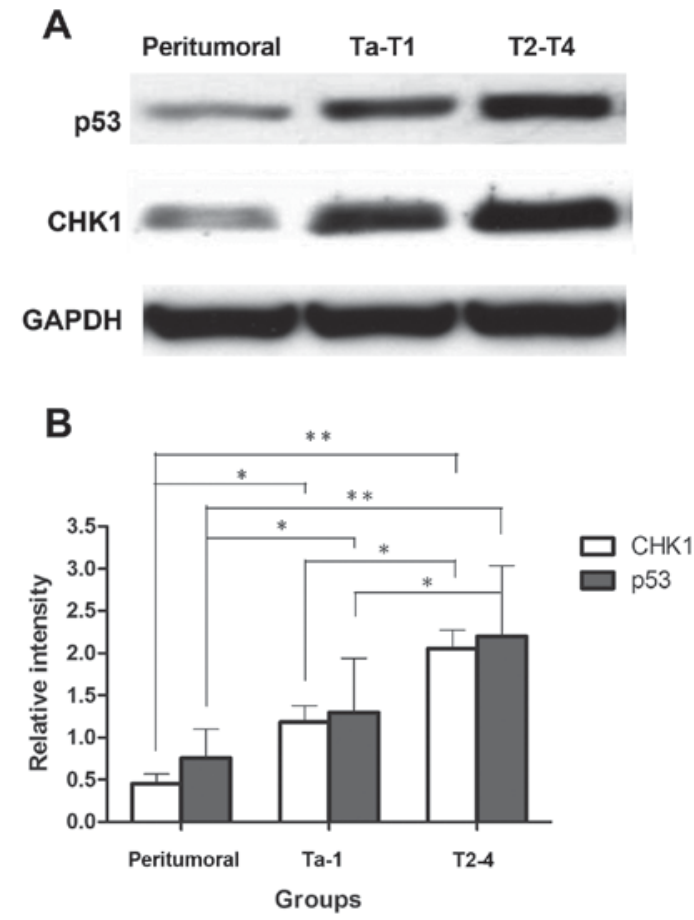

Figure 2. Western blot analysis of CHK1 and p53 expression in peritumoral, Ta-T1, and T2-T4 BUC groups, respectively. Four independent repeated tests were done in each group by western blot analysis, the intensity of each band was quantified by gray-scale analysis, and the representative bands were selected to be demonstrated. (A) The expression of CHK1 and p53 proteins presented an increasing tendency from peritumoral to Ta-T1 and T2-T4 groups, GAPDH served as an internal control. (B) The signal of Western blotting was quantified using gray-scale analysis software and data was normalized to GAPDH. Data represent the mean \pm SD of 4 independent experiments. ${ }^{*} \mathrm{P}<0.05,{ }^{* *} \mathrm{P}<0.01$. CHK1, checkpoint kinase 1.

in the 5-year survival rate than other sub-groups $\left(\chi^{2}=18.97\right.$, $\mathrm{P}<0.001$ ) (Fig. 4A). However, in MIBC group, the 5-year 
A

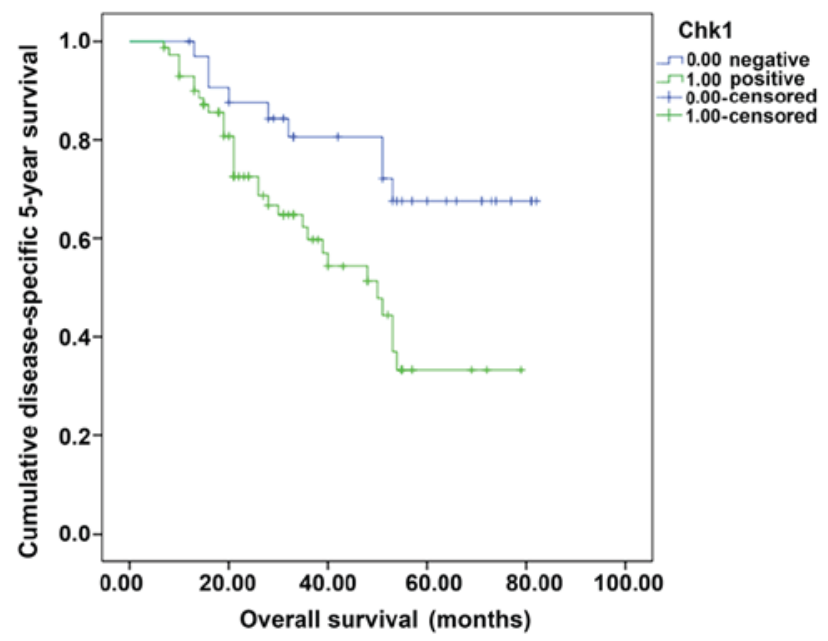

B

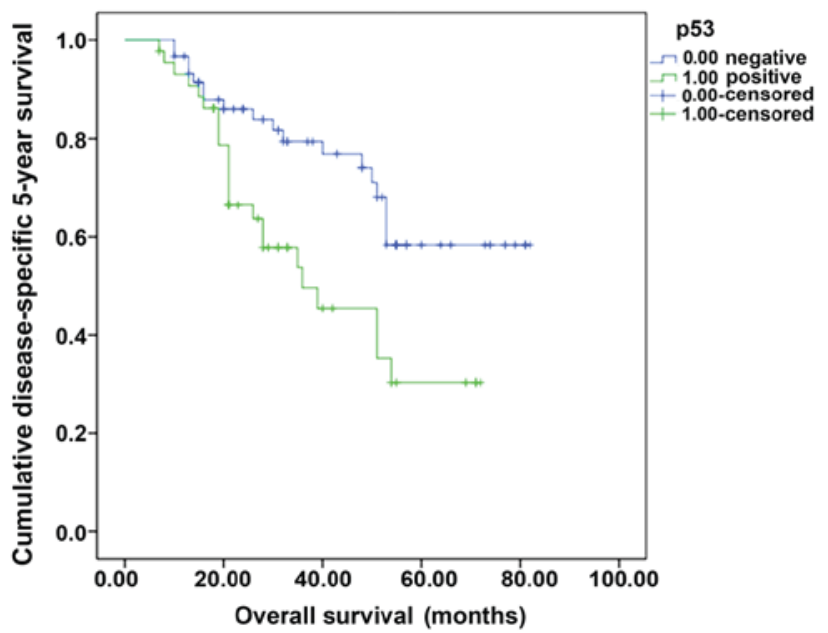

Figure 3. The 5-year survival rate of CHK1 or p53 in BUC. (A) The difference between CHK1-positive (81 cases) and -negative (29 cases) was statistically significant $\left(\chi^{2}=6.98, P=0.008\right)$. (B) Similarly results was indicated between p53-positive $(58$ cases $)$ and - negative $(52$ cases $)\left(\chi^{2}=7.63\right.$, $\left.P=0.006\right)$. $C H K 1$, checkpoint kinase 1; BUC, bladder urothelial carcinoma.

A

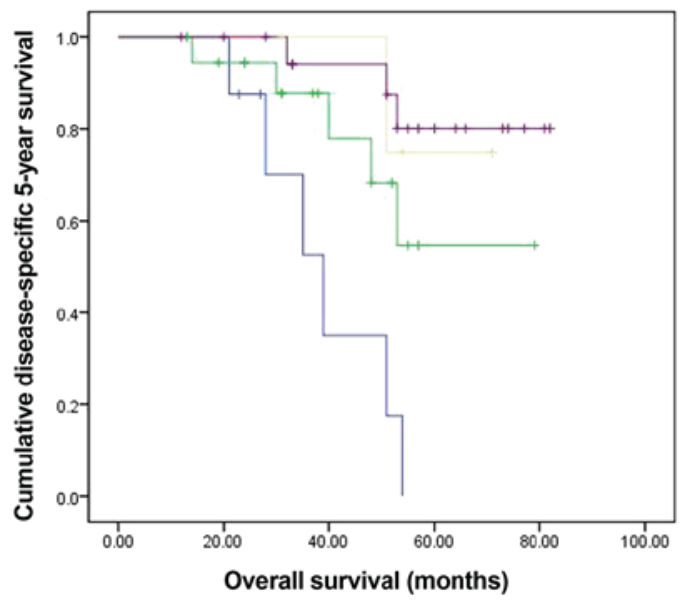

B

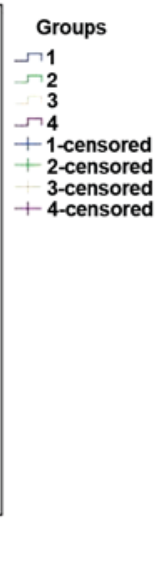

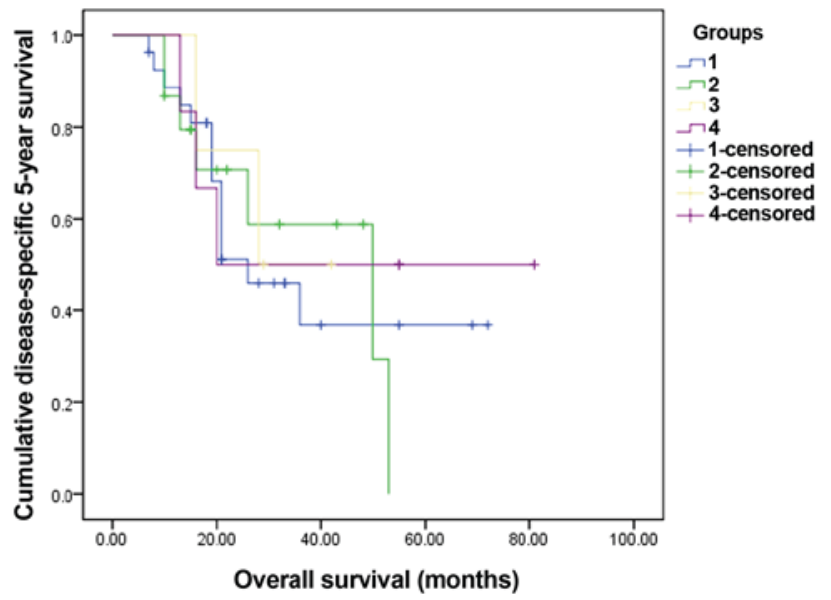

Figure 4. The 5-year survival rate of sub-groups in NMIBC (50 cases) and MIBC (60 cases). Four sub-groups were divided according to the markers: group 1, CHK1 positive and p53 positive (NMIBC, 8 cases; MIBC, 27 cases); group 2, CHK1 positive and p53 negative (NMIBC, 24 cases; MIBC, 18 cases); group 3, CHK1 negative and p53 positive (NMIBC, 9 cases; MIBC, 9 cases); group 4, both CHK1 and p53 were negative (NMIBC, 9 cases; MIBC, 6 cases). Significant differences were checked in NMIBC $\left(\chi^{2}=18.97, \mathrm{P}<0.001\right)(\mathrm{A})$ yet in MIBC no significant differences $\left(\chi^{2}=0.34, \mathrm{P}=0.952\right)(\mathrm{B})$. CHK1, checkpoint kinase 1 ; NMIBC, non-muscle-invasive bladder cancer; MIBC, muscle-invasive bladder cancer.

survival rate did not had significant difference among the sub-groups $\left(\chi^{2}=0.34, \mathrm{P}=0.952\right)$ (Fig. 4B). Furthermore, we checked the multivariate Cox analyses to elucidate the prognostic effects of the biological marker, the data revealed that only metastasis was an independent prognostic risk factor $(\mathrm{P}<0.001)$ (Table IV).

\section{Discussion}

DNA damage response is a complex signaling network, which includes cell cycle checkpoints and DNA damage and repair pathways. DNA damage induces G1/S arrest or G2/M arrest to prevent the cells carrying damaged chromosomes from progressing into mitosis. The $\mathrm{G} 2 / \mathrm{M}$ arrest is principally mediated by the activation of serine/threonine kinase CHK1, whereas the G1/S checkpoint is primarily mediated through the tumor suppressor p53 (25). p53 is known to enhance the CHK1-mediated G2/M checkpoint activation induced by chemotherapeutics. The downregulation of the molecule can be prevented by inhibitors against JAK2, BCR/ABL, or the PI3K/Akt pathway (26).

The current study indicated the expression of CHK1 between BUC and peritumoral tissues differed significantly $(\mathrm{P}<0.05)$. CHK1 exhibited an increasing deterioration of the pathological grading and clinical staging. Survival analysis indicated a negative prognosis in CHK1 overexpression cases. On the other hand, the evaluation of p53 in BUC specimens retrieved results similar to that of $\mathrm{CHK} 1$, demonstrating a remarkable difference between BUC and peritumoral tissues $(\mathrm{P}<0.05)$, and growing trend with an elevated degree of BUC malignancy. To further confirm the results, we assessed the correlation between CHK1 and p53 in BUC; the close 
Table IV. Univariate and multivariate Cox regression analyses estimating the associations of CHK1 and p53 expression with patient survival.

\begin{tabular}{|c|c|c|c|c|c|c|}
\hline Characteristic & Crude HR & $95 \% \mathrm{CI}$ & P-value & Adjust HR & $95 \% \mathrm{CI}$ & P-value \\
\hline \multicolumn{7}{|l|}{ Age, years } \\
\hline$<63$ & 1 & & & & & \\
\hline$\geq 63$ & 2.45 & $1.28-4.67$ & 0.006 & & & \\
\hline \multicolumn{7}{|l|}{ Pathologic T } \\
\hline $\mathrm{Ta}-\mathrm{T} 1$ & 1 & & & & & \\
\hline $\mathrm{T} 2-\mathrm{T} 4$ & 2.40 & $1.30-4.43$ & 0.005 & & & \\
\hline \multicolumn{7}{|c|}{ Lymphatic metastasis } \\
\hline Negative & 1 & & & 1 & & \\
\hline Positive & 5.45 & $2.80-10.61$ & $<0.001$ & 4.75 & $2.39-9.44$ & $<0.001$ \\
\hline \multicolumn{7}{|l|}{$\mathrm{p} 53$} \\
\hline Negative & 1 & & & & & \\
\hline Positive & 2.07 & $1.13-3.79$ & 0.019 & & & \\
\hline \multicolumn{7}{|l|}{ CHK1 } \\
\hline Negative & 1 & & & & & \\
\hline Positive & 2.76 & $1.31-5.81$ & 0.007 & & & \\
\hline
\end{tabular}

HR, hazard ratio; CI, confidence interval; BUC, bladder urothelial carcinoma; CHK1, checkpoint kinase 1.

correlation of CHK1 and p53 indicated synergistic interaction in BUC progression. These data confirmed the evidence that both CHK1 and p53 were associated with BUC and could serve as putative targets for treating BUC. This conclusion was in agreement with previous studies with respect to CHK1 and p53 in cancer research $(14,25,27)$. However, the precise underlying mechanism is not yet elucidated.

The ATM/CHK signaling requires p53 to mediate the physiological function (28). In ATM-CHK-p53 axis, the enhanced proliferative pressure and genomic instability of both precancerous lesions and cancers generate a considerable amount of spontaneous DNA damage. This accumulated DNA damage induces cell cycle arrest, senescence, and apoptosis via p53 activation. Followed by full activation of the replication stress response, the activated ATM/ATR phosphorylates CHK1 resulting in the activation of downstream effector molecules, including p53. In another pathway, p53 putatively regulates $\mathrm{CHK} 1$ in a positive feedback mechanism. For example, Bernard et al reported that phosphorylation of CHK1 on Ser317 was regulated by p53; thus, p53 may act as a molecular 'on/off' switch for the phosphorylation of CHK1 on Ser317 (29).

To confirm the deduction above, we checked the 5-year survival rate of sub-groups in NMIBC and MIBC groups (Fig. 4A and B). In NMIBC group, the survival images indicated CHK1 positive and p53 positive sub-groups had the worst prognosis, CHK1 negative and $\mathrm{p} 53$ negative sub-groups had the best prognosis, and the other sub-groups were in middle. The results confirmed CHK1 and p53 could be prognostic factors in patients suffering from BUC, CHK1 and p53 probably had synergistic interaction in CHK1 positive and $\mathrm{p} 53$ positive cases, which probably indicated poor prognosis. However, in MIBC group, there was no significant difference in the sub-groups. This result might be due to the limited sample and a high mortality in MIBC. In the multivariate Cox analyses, we found metastasis was an independent prognostic risk factor, other factors including sex, age, tumor diameter, single/multiple sites, histological grade, clinical pathological staging, CHK1, p53, patients with incipient/recurrence were not independent prognostic risk factors. The results indicated that an effective biomarker to predict tumor metastasis of BUC.

Our study had several limitations. Firstly, as there was small sample size in NMIBC and MIBC, we did not do EORTC table to confirm the markers' validation in further; then, the small cases in each sub-group in NMIBC or MIBC group, probably increased the error in 5-year survival rate analysis. So, we expected to collect more samples and finish the analysis in further research.

In summary, those results indicated CHK1 and p53 in BUC, especially in NMIBC, could be regarded as the potential chemotherapeutic target. And further research about the underlying mechanism remains needed.

As delineated above, the present study, for the first time, assessed the association of CHK1 and p53 in BUC, suggesting potential prognosis or therapeutic target in the progression of BUC.

\section{Acknowledgements}

The authors would like to thank Jan-Guo Feng for their technical assistance.

\section{References}

1. Miller KD, Siegel RL, Lin CC, Mariotto AB, Kramer JL, Rowland JH, Stein KD, Alteri R and Jemal A: Cancer treatment and survivorship statistics, 2016. CA Cancer J Clin 66: 271-289, 2016.

2. Prasad SM, Decastro GJ and Steinberg GD; Medscape: URothelial carcinoma of the bladder: Definition, treatment and future efforts. Nat Rev Urol 8: 631-642, 2011. 
3. Abdollah F, Gandaglia G, Thuret R, Schmitges J, Tian Z, Jeldres C, Passoni NM, Briganti A, Shariat SF, Perrotte P, et al: Incidence, survival and mortality rates of stage-specific bladder cancer in united states: A trend analysis. Cancer Epidemiol 37: 219-225, 2013.

4. Bishr M, Lattouf JB, Latour M and Saad F: Tumour stage on re-staging transurethral resection predicts recurrence anad progression-free survival of patients with high-risk non-muscle invasive bladder cancer. Can Urol Assoc J 8: E306-E310, 2014.

5. Yafi FA, Aprikian AG, Chin JL, Fradet Y, Izawa J, Estey E, Fairey A, Rendon R, Cagiannos I, Lacombe L, et al: Contemporary outcomes of 2287 patients with bladder cancer who were treated with radical cystectomy: A canadian multicentre experience. BJU Int 108: 539-545, 2011.

6. Soloway MS: Bladder cancer: Lack of progress in bladder cancer-what are the obstacles? Nat Rev Urol 10: 5-6, 2013.

7. Nedjadi T, Al-Maghrabi J, Assidi M, Dallol A, Al-Kattabi H, Chaudhary A, Al-Sayyad A, Al-Ammari A, Abuzenadah A Buhmeida A and Al-Qahtani M: Prognostic value of HER2 status in bladder transitional cell carcinoma revealed by both IHC and BDISH techniques. BMC Cancer 16: 653, 2016.

8. Wang ZY, Zhang W, Yang JJ, Song DK, Wei JX and Gao S: Association of thymosin $\beta 4$ expression with clinicopathological parameters and clinical outcomes of bladder cancer patients. Neoplasma 63: 991-998, 2016.

9. Zhou J, Dong D, Cheng R, Wang Y, Jiang S, Zhu Y, Fan L, Mao X, Gui Y, Li Z, et al: Aberrant expression of KPNA2 is associated with a poor prognosis and contributes to OCT4 nuclear transportation in bladder cancer. Oncotarget 7: 72767-72776, 2016.

10. van der Heijden AG, Mengual L, Lozano JJ, Ingelmo-Torres M, Ribal MJ, Fernandez PL, Oosterwijk E, Schalken JA, Alcaraz JA, Alcaraz A and Witjes JA: A five-gene expression signature to predict progression in T1G3 bladder cancer. Eur J Cancer 64: 127-136, 2016.

11. Pignot G, Cizeron-Clairac G, Vacher S, Susini A, Tozlu S, Vieillefond A, Zerbib M, Lidereau R, Debre B, Amsellem-Ouazana D and Bieche I: microRNA expression profile in a large series of bladder tumors: identification of a 3-miRNA signature associated with aggressiveness of muscle-invasive bladder cancer. Int J Cancer 132: 2479-2491, 2013

12. Segersten U, Spector Y, Goren Y, Tabak S and Malmström PU: The role of microRNA profiling in prognosticating progression in Ta and T1 urinary bladder cancer. Urol Oncol 32: 613-618, 2014.

13. Goto H, Kasahara K and Inagaki M: Novel insights into Chk1 regulation by phosphorylation. Cell Struct Funct 40: 43-50, 2015.

14. Toledo LI, Murga M and Fernandez-Capetillo O: Targeting ATR and Chk1 kinases for cancer treatment: A new model for new (and old) drugs. Mol Oncol 5: 368-373, 2011

15. Petermann E, Maya-Mendoza A, Zachos G, Gillespie DA, Jackson DA and Caldecott $\mathrm{KW}$ : Chk1 requirement for high global rates of replication fork progression during normal vertebrate S phase. Mol Cell Biol 26: 3319-3326, 2006.

16. Petermann E, Woodcock M and Helleday T: Chk1 promotes replication fork progression by controlling replication initiation. Proc Natl Acad Sci USA 107: 16090-16095, 2010.
17. Herman-Antosiewicz A, Stan SD, Hahm ER, Xiao D and Singh SV: Activation of a novel ataxia-telangiectasia mutated and $\mathrm{Rad} 3 \mathrm{related} / \mathrm{checkpoint} \mathrm{kinase} \mathrm{1-dependent} \mathrm{prometaphase}$ checkpoint in cancer cells by diallyl trisulfide, a promising cancer chemopreventive constituent of processed garlic. Mol Cancer Ther 6: 1249-1261, 2007.

18. Myers K, Gagou ME, Zuazua-Villar P, Rodriguez R and Meuth M: ATR and Chk1 suppress a caspase-3-dependent apoptotic response following DNA replication stress. PLoS Genet 5 : e1000324, 2009.

19. Wasylishen AR and Lozano G: Attenuating the p53 pathway in human cancers: many means to the same end. Cold Spring Harb Perspect Med 6: a026211, 2016

20. Vazquez A, Bond EE, Levine AJ and Bond GL: The genetics of the p53 pathway, apoptosis and cancer therapy. Nat Rev Drug Discov 7: 979-987, 2008.

21. Williams AB and Schumacher B: p53 in the DNA-Damage-Repair Process. Cold Spring Harb Perspect Med 6, 2016.

22. Freed-Pastor WA and Prives C: Mutant p53: One name, many proteins. Genes Dev 26: 1268-1286, 2012

23. Humphrey PA, Moch H, Cubilla AL, Ulbright TM and Reuter VE: The 2016 WHO classification of tumours of the urinary system and male genital organs-part B: Prostate and bladder tumours. Eur Urol 70: 106-119, 2016

24. Rath S, Connors JM, Dolman PJ, Rootman J, Rootman DB and White VA: Comparison of American Joint Committee on Cancer TNM-based staging system (7th edition) and Ann Arbor Classification for predicting outcome in ocular adnexal lymphoma. Orbit 33: 23-28, 2014.

25. Drayton RM and Catto JW: Molecular mechanisms of cisplatin resistance in bladder cancer. Expert Rev Anticancer Ther 12: 271-281, 2012.

26. Li CC, Yang JC, Lu MC, Lee CL, Peng CY, Hsu WY, Dai YH, Chang FR, Zhang DY, Wu WJ and Wu YC: ATR-Chk1 signaling inhibition as a therapeutic strategy to enhance cisplatin chemosensitivity in urothelial bladder cancer. Oncotarget 7: 1947-1959, 2016.

27. Umezawa Y, Kurosu T, Akiyama H, Wu N, Nogami A, Nagao T and Miura O: Down regulation of Chk1 by p53 plays a role in synergistic induction of apoptosis by chemotherapeutics and inhibitors for Jak2 or BCR/ABL in hematopoietic cells. Oncotarget 7: 44448-44461, 2016.

28. Manic G, Obrist F, Sistigu A and Vitale I: Trial Watch: Targeting ATM-CHK2 and ATR-CHK1 pathways for anticancer therapy. Mol Cell Oncol 2: e1012976, 2015.

29. Bernard JJ, Lou YR, Peng QY, Li T, Conney AH and Lu YP: Inverse relationship between p53 and phospho-Chk1 (Ser317) protein expression in UVB-induced skin tumors in SKH-1 mice. Exp Mol Pathol 96: 126-131, 2014. 